\title{
Visual short-term memory for sequential arrays
}

\author{
ARJUN KUMAR and YUHONG JIANG \\ Harvard University, Cambridge, Massachusetts
}

\begin{abstract}
The capacity of visual short-term memory (VSTM) for a single visual display has been investigated in past research, but VSTM for multiple sequential arrays has been explored only recently. In this study, we investigate the capacity of VSTM across two sequential arrays separated by a variable stimulus onset asynchrony (SOA). VSTM for spatial locations (Experiment 1), colors (Experiments 2-4), orientations (Experiments 3 and 4), and conjunction of color and orientation (Experiment 4) were tested, with the SOA across the two sequential arrays varying from 100 to 1,500 msec. We find that VSTM for the trailing array is much better than VSTM for the leading array, but when averaged across the two arrays VSTM has a constant capacity independent of the SOA. We suggest that multiple displays compete for retention in VSTM and that separating information into two temporally discrete groups does not enhance the overall capacity of VSTM.
\end{abstract}

Visual short-term memory (VSTM) is the component of working memory that retains visual information for up to several seconds (Logie, 1995). It creates temporal continuity in a constantly changing visual environment, yet it has severe capacity limitations. Only four objects or six spatial locations can be retained at once in VSTM (Alvarez \& Cavanagh, 2004; Cowan, 2001; Jiang, Olson, \& Chun, 2000; Luck \& Vogel, 1997; Pashler, 1988; Phillips, 1974). This small capacity places a high demand on the visual system to retain only the most important information from a scene in VSTM.

Surprisingly, research efforts in the past 5 years have focused primarily on the representation of a single visual display in memory. Nevertheless, in many everyday activities visual information not only occupies space but also evolves over time. Driving requires the constant updating of information gathered from many instances, crossing a busy street requires memories of what is on one's left and right, and even the simplest social interactions depend on retaining information across space and time. A natural visual input rarely appears in temporal isolation but rather is imbedded in the preceding and following scenes. Because the natural environment presents successive inputs, investigating memory for a single display cannot explain the representation of the real world in VSTM; we must also investigate memory for successive inputs. Past studies in this area have shown that successive inputs may interact by competing for space in a limited-capacity VSTM (Jiang, 2004), by producing proactive or retroactive interference (Broadbent \& Broad-

This study forms part of A.K.'s senior honors thesis, submitted to Harvard University. It was supported by NSF Grant 0345525 and a Milton Fund grant. We thank James Brockmole, Patrick Cavanagh, Kyle Cave, Robert Greene, and Steven Luck for comments and suggestions. Correspondence concerning this article should be addressed to Y. Jiang, 33 Kirkland Street, WJH 820, Cambridge, MA 02138 (e-mail: arjun. kumar@post.harvard.edu or yuhong@wjh.harvard.edu). bent, 1981; Brockmole, Wang, \& Irwin, 2002; Jiang \& Kumar, 2004), or by becoming integrated into a combined display (Brockmole et al., 2002). In this study, we examine how separating information into two temporal arrays affects the overall capacity of VSTM. In the following introduction, we shall first briefly review past research on VSTM for a single display and then discuss how sequential presentation affects VSTM capacity.

\section{VSTM for a Single Display}

A briefly presented array of items is first held in iconic memory (Irwin, 1991; Loftus, Duncan, \& Gehrig, 1992; Phillips, 1974; Sperling, 1960). As the icon decays, a small subset of visual information is transferred to VSTM. The capacity of VSTM is severely limited to approximately four items (Luck \& Vogel, 1997; Pashler, 1988) and six spatial locations (Jiang et al., 2000). This small capacity varies little with variation in features (e.g., colors, orientations, sizes; Luck \& Vogel, 1997) or familiarity (e.g., right-side-up letters vs. upside-down letters; Pashler, 1988). However, the items occupying this capacity may vary in complexity, according to theories of object-based representation (D. Lee \& Chun, 2001; Luck \& Vogel, 1997; Raffone \& Wolters, 2001; Walker \& Davies, 2003) or perceptual grouping (Jiang, Chun, \& Olson, 2004; Woodman, Vecera, \& Luck, 2003). These theories show that VSTM can retain four visual objects, each characterized by a conjunction of several features (e.g., color, orientation, and size), just as well as it can hold four visual objects with only one feature per object. This increase in capacity for features is explained primarily by the chunking of distinct features into unified objects (Luck \& Vogel, 1997; Vogel, Woodman, \& Luck, 2001) and secondarily by the heterogeneity of the to-beremembered information (e.g., memory for four different kinds of features - color, orientation, size, and textureis better than memory for four features of the same kind; Delvenne \& Bruyer, 2004; Olson \& Jiang, 2002; Wheeler 
\& Treisman, 2002; Xu, 2002a, 2002b). In addition to object-level chunking, multiple items can be grouped on the basis of depth (Xu \& Nakayama, 2003), or they can be remembered as a single, complex pattern rather than as several isolated items (Jiang et al., 2000).

\section{VSTM for Sequential Arrays}

The influential study by Luck and Vogel (1997) motivated several investigations on VSTM of a single static display. These studies, however, did not address how multiple arrays are remembered. To answer this question, we must go back two decades: Broadbent and Broadbent (1981) presented subjects with four sequential visual patterns followed immediately by a recognition test in reverse order (i.e., the fourth pattern was tested first). They observed a recency effect in that memory for the fourth pattern was good but memory for the first three was poor.

Related studies in this area have shown that when subjects see a series of rapidly presented displays, with a short (100-msec) interval between the members of any consecutive pair, each display interferes with memory for the previous display via both sensory and conceptual masking (see, e.g., Potter, 1976). Actively searching for a particular display in such a series improves memory accuracy for the attended display, and at intervals beyond $100 \mathrm{msec}$ sensory masking is greatly reduced. These studies suggest that after a visual display appears it takes 200-300 msec of focused attention for VSTM to consolidate the display into a stable representation (Jolicœur \& Dell'Acqua, 1998; Potter, Staub, \& O'Connor, 2002; Vogel, Woodman, \& Luck, in press).

The studies summarized above show that sequential displays presented close together interfere with one another, and the degree of interference depends partly on the stimulus onset asynchrony (SOA) between the two displays. At extremely short $(<100-\mathrm{msec})$ SOAs, the two arrays are integrated in iconic memory and are perceived as a single display (Di Lollo, 1980). At SOAs of 100-300 msec, however, the second array masks the first, leading to a weak percept of the first array. Then, as SOA increases beyond $500 \mathrm{msec}$, the two arrays start to be perceived as separate displays (Jiang \& Kumar, 2004). They are integrated only under some task conditions (Brockmole et al., 2002; Jiang, Kumar, \& Vickery, 2005). Given that subjects retain separate VSTM representations for the two arrays at long SOAs, we asked whether dividing information into two temporally discrete arrays would enhance the overall capacity of VSTM.

\section{Grouping and VSTM}

In verbal short-term memory, subgrouping items by inserting a temporal pause between them (e.g., in telephone numbers, such as 617-450-2343) facilitates memory (Baddeley, 1986), and in VSTM subgrouping items by presenting them in different depth planes enhances overall memory capacity (Xu \& Nakayama, 2003). Be- cause temporal segregation is another powerful grouping cue in visual perception (Alais, Blake, \& Lee, 1998; Blake \& Yang, 1997; Jiang, Chun, \& Marks, 2002; S. H. Lee \& Blake, 1999; Usher \& Donnelly, 1998), dividing information across several arrays rather than presenting it all at once may enhance VSTM. The present study is our first broad attempt to compare VSTM capacity for sequential and simultaneous presentations.

\section{Overview of the Experiments}

Two arrays, each containing several items (e.g., five dot locations), were presented in this study. The SOA between the two arrays was short $(27 \mathrm{msec})$, intermediate (127-527 msec), or long (1,027 msec or longer). When the SOA was $27 \mathrm{msec}$, the two arrays were integrated in iconic memory, leading to the percept that all items were presented simultaneously. ${ }^{1}$ When the SOA was long, the two arrays were clearly perceived as two, so they competed for the limited capacity of VSTM. At intermediate SOAs (e.g., 127-227 msec), Array 2 interacted with Array 1 by producing sensory masking (Loftus et al., 1992), conceptual masking (Jiang, 2004; Jolicœur \& Dell'Acqua, 1998; Potter, 1976; Vogel et al., in press), or integration (Brockmole, Irwin, \& Wang, 2003; Brockmole et al., 2002). Thus, results obtained for these SOAs would not offer a pure measure of VSTM capacity; ${ }^{2}$ they were included for completeness of the time-course information. Conclusions will be drawn primarily on the basis of short and long SOAs. Of interest is whether the total amount of information held in VSTM depends on the SOA. VSTM for spatial locations, colors, different features (colors and orientations), and the conjunction of color and orientation were tested in separate experiments.

\section{EXPERIMENT 1 VSTM for Spatial Locations}

In this experiment, we addressed whether or not dividing spatial information into temporally discrete sets increases VSTM capacity for spatial locations. We presented subjects with two memory arrays, each containing five dot locations, separated by a variable SOA. One second after the presentation of Array 2, a probe array with two items was presented. The subjects decided which of the two items in the probe array matched an item from either memory array. A similar manipulation of array and SOA has been used in the studies of Brockmole and colleagues, although with a different theoretical focus (Brockmole et al., 2003; Brockmole et al., 2002).

If separating spatial information into temporally discrete groups allows VSTM to retain more information, performance should be better when the SOA between the two memory arrays is long, leading to the percept of two sequential arrays. Conversely, if VSTM has a fixed capacity limit for which the two arrays compete, overall performance should be comparable whether subjects perceive the arrays as sequential or as simultaneous. 


\section{Method}

Subjects. Twelve subjects from the Boston area, ranging in age from 18 to 35 years, participated in the experiment for payment. All the subjects had normal or corrected-to-normal visual acuity.

Presentation sequence. Each subject was tested individually in a dimly lit room, with a 19 -in. computer monitor approximately $57 \mathrm{~cm}$ away. (At this distance, $1 \mathrm{~cm}$ corresponds to $1^{\circ}$ of visual angle.) Each trial started with a visible, empty $5 \times 5\left(16^{\circ} \times 16^{\circ}\right)$ grid with a gray background, presented for $400 \mathrm{msec}$. Then, a memory array of five white dots $\left(0.8^{\circ}\right.$ in diameter $)$ was presented for $27 \mathrm{msec}$ at randomly selected locations within the grid. After a variable SOA, the second memory array of five other dots was presented at new locations, for $27 \mathrm{msec}$. After a 1,000-msec retention interval, a probe array containing two dots was presented until the subject made a response. Since each array contained fewer than six items, each array alone fell within the spatial capacity of VSTM. Figure 1 shows a schematic sample of the display.

Materials. The probe array showed two dots, each with a small "1" or " 2 " on it. One of these dots was in the same location as a randomly determined dot from either Array 1 or Array 2, and the other was in a previously unoccupied location. The numeral that marked each dot was randomly assigned. The subjects identified which of the two dots they had previously seen by pressing the " 1 " or the " 2 " key on the computer keyboard. They did not have to identify which array the target dot was from, just which one they had seen on either array. The number that a subject pressed should correspond to

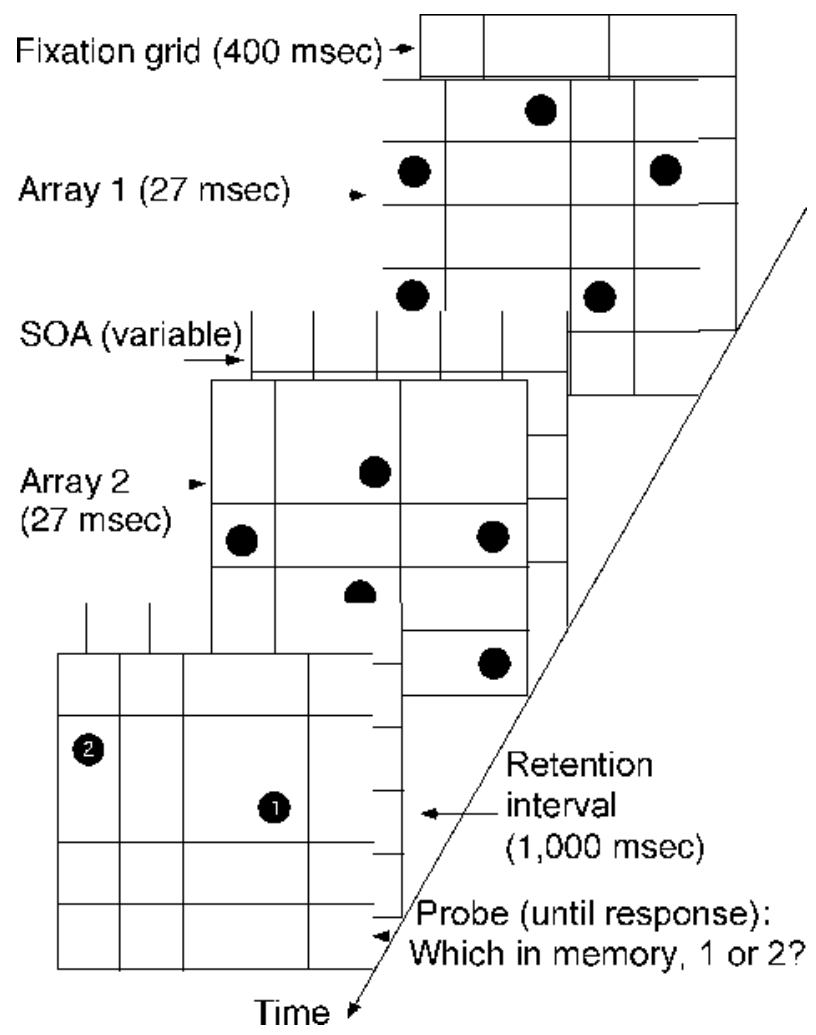

Figure 1. A schematic sample of the presentation sequence tested in Experiment 1. Subjects were told to remember the locations occupied by the dots in Arrays 1 and 2 and to judge which of two probe dots matched the locations in their memory. One probe was at a previously blank location, and the other matched the location of a dot in either Array 1 or Array 2, equally often. the number marking the matching probe dot. Accuracy feedback was provided immediately after each response. To motivate performance, a score was kept, and it appeared after each trial. The subjects gained two points for each correct response and lost one point for each incorrect response.

Each subject completed 20 practice trials and 280 experimental trials, divided into four blocks of 70 trials each. The SOA between the two arrays was $27,127,227,527$, or $1,227 \mathrm{msec}$, with each SOA randomly assigned to one fifth of all trials.

\section{Results}

We calculated accuracy for each SOA condition and array. For the array variable, one of the two probe dots matched either Array 1 or Array 2. This gave us a measure of memory accuracy for positions drawn from the two arrays separately. Figure 2 shows the mean across all subjects.

A repeated measures analysis of variance (ANOVA) on array (Array 1 vs. Array 2) and SOA (27 vs. 127 vs. 227 vs. 527 vs. $1,227 \mathrm{msec}$ ) revealed a significant main effect of array $[F(1,11)=35.03, p<.001]$, with memory for Array 2 being better than that for Array 1. The main effect of SOA was not significant $(F<1)$, but the interaction between array and SOA was $[F(4,44)=2.69$, $p<.05]$. The lack of the SOA main effect suggests that, pooled across the two arrays, overall performance was unaffected by SOA (this conclusion held even when ANOVAs were conducted on Array 1 and Array 2 separately; $p$ values $>.10$ ). However, SOA modulated the tradeoff between the two arrays.

When the SOA was $27 \mathrm{msec}$, the two arrays were integrated as one in iconic memory, at which point memory for Array 1 and that for Array 2 were not significantly different from each other $[t(11)=1.27, p>.20]$. However, when the SOA was $1,227 \mathrm{msec}$, the two arrays were perceived as two separate displays, at which point accuracy for Array 2 was significantly higher than accuracy for Array $1[t(11)=4.55, p<.005]$. However, when pooled across the two arrays, the overall accuracy was comparable between an SOA of $27 \mathrm{msec}(69 \%)$ and an SOA of $1,227 \mathrm{msec}(70 \%)[t(11)=0.58, p>.50]$.

\section{Discussion}

When spatial information was distributed across two sequential arrays, there was a clear memory advantage of the trailing array over the leading array. Accuracy for determining whether a probe dot matched a dot in the visual memory was higher for the second array than for the first. This observation suggests that two temporally segregated arrays compete for the limited capacity of VSTM and that Array 2 has an advantage for being retained (see also Brockmole et al., 2002; Jiang, 2004; Jiang et al., 2005). Moreover, when pooled across the two arrays, accuracy in the VSTM task was comparable whether the two arrays were sensorily integrated into one or perceived as separate displays. This suggests that dividing spatial locations into two temporal groups does not enhance the overall capacity of VSTM. Both observationsof the competition between the two arrays and of the 


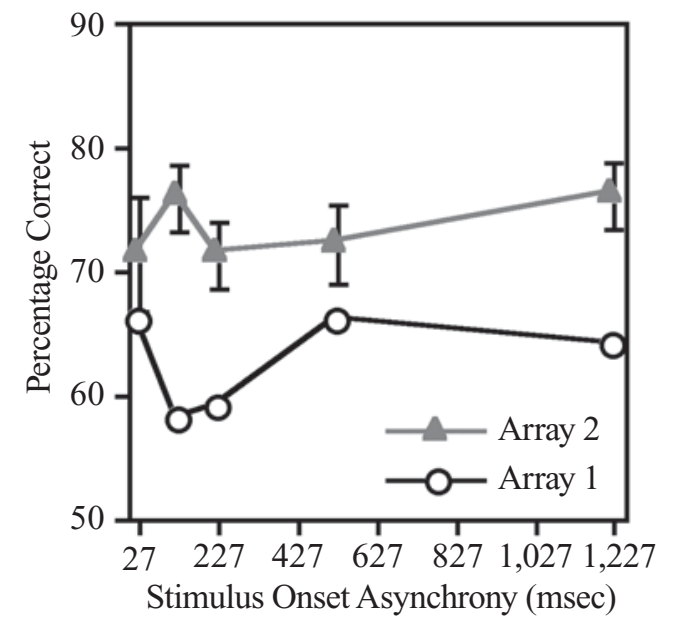

Figure 2. Results from Experiment 1. Accuracy was higher when memory for Array 2 was probed than when that for Array 1 was probed, but the overall accuracy was comparable between simultaneous VSTM (SOA $=27 \mathrm{msec}$ ) and sequential VSTM (other SOAs). The error bars show standard errors of the differences between Arrays 1 and 2.

constant overall capacity—place significant theoretical constraints on the representation of temporal sequences in VSTM.

The constant overall capacity of VSTM suggests that temporal segregation has a restricted role in enhancing memory. This can be contrasted against spatial segregation and feature conjunction, both of which can significantly enhance overall VSTM capacity. As was reviewed earlier, separating visual information into two depth planes (Xu \& Nakayama, 2003) increases the total amount of information held in VSTM. In addition, conjoining separate features into a single object dramatically increases the total number of features retained in VSTM (Luck \& Vogel, 1997).

The failure to enhance overall VSTM capacity through a temporal segregation cue suggests that not all perceptual grouping cues are equally effective. That is, certain ways of chunking items together, such as object-based feature conjunction and depth grouping, are more effective than other cues in enhancing VSTM. Temporal segregation is not an effective cue. Presenting items in two temporal sequences does not increase the capacity of VSTM. Instead, a different proportion of VSTM capacity is allocated to each array, with more allocated to the trailing array.

Why was there an advantage for the trailing array relative to the leading array? Poorer memory for Array 1 may have been due to memory decay. As the SOA increased, the interval between the offset of Array 1 and the probe also increased, from $1,000 \mathrm{msec}(\mathrm{SOA}=27 \mathrm{msec})$ to $2,200 \mathrm{msec}(\mathrm{SOA}=1,227 \mathrm{msec})$. Memory for Array 1 may have decayed during that period of time. In contrast, the interval between the offset of Array 2 and the probe was held constant $(1,000 \mathrm{msec})$ across various SOAs. Thus, VSTM for Array 2 would have decayed less than that for Array 1, particularly at longer SOAs.
Although memory decay contributed to poor retention of Array 1, it is unlikely that it was the only factor. Consider two conditions: (1) When the SOA was $127 \mathrm{msec}$, Array 1 had decayed for $1,100 \mathrm{msec}$ by the time the probe array was presented and its memory accuracy was $58 \%$; and (2) when the SOA was $1,227 \mathrm{msec}$, Array 2 had decayed for $1,000 \mathrm{msec}$ by the time the probe array was presented and its memory accuracy was $76 \%$. These two levels of performance are very different $[t(11)=$ $5.40, p<.001]$, yet the decay periods are similar. Thus, decay cannot completely account for the difference in performance across arrays. The second important factor contributing to Array 1's poor accuracy was the competition between the two arrays. When Array 2 entered VSTM, information about Array 1 was pushed out, leading to an advantage for Array 2. In light of this hypothesis, Jiang (2004) asked subjects to ignore Array 2 and retain only Array 1 in VSTM. Under this condition, memory for Array 1 was comparable across short and long SOAs.

In summary, results from Experiment 1 are consistent with the idea that separating spatial information into two sequential arrays does not facilitate the overall capacity of VSTM. The two arrays compete for the limited capacity, with Array 2 winning the competition as the SOA between the two arrays increases. These results are inconsistent with the view that perceptual segregation in general enhances VSTM. They suggest instead that VSTM capacity is insensitive to the presentation mode, whether it is sequential or simultaneous.

\section{EXPERIMENT 2 VSTM for Colors}

Experiment 1 demonstrated that VSTM capacity is independent of the mode of presentation. The total amount of spatial information retained in VSTM is the same whether such information is perceived as a single array or as temporally separated. In Experiment 2, we investigated VSTM for sequential arrays, with the important difference that subjects were required to remember colors instead of spatial locations. VSTM for colors is more limited than VSTM for spatial locations; only about four colors can be retained when presented simultaneously (Luck \& Vogel, 1997; Pashler, 1988), as opposed to about six spatial locations (Jiang et al., 2000). Because the visual system processes color and location information separately (Livingstone \& Hubel, 1988; Mishkin \& Ungerleider, 1983) and because VSTM has different capacities for colors and for locations, one cannot simply assume that temporal segregation would have similar effects on VSTM for colors and for locations.

To find out whether temporal segregation enhances VSTM for colors, we tested subjects in a design similar to that of Experiment 1, except that the subjects were told to remember colors. Three colored circles were briefly presented in Array 1, followed by a variable SOA, and three differently colored circles were presented in Array 2. After a retention interval of $1 \mathrm{sec}$, the subjects were shown a single probe and had to determine whether the 
probe color matched any of the previously presented colors. Of interest was how the two arrays competed for VSTM capacity and whether the overall capacity for colors was independent of the SOA. Figure 3 shows a schematic sample of a trial.

\section{Method}

Subjects. Twelve subjects participated in this experiment for payment. All had normal or corrected-to-normal visual acuity and normal color vision, which was verified with a color blindness test.

Trial sequence. The temporal sequence on the computer screen was similar to that of Experiment 1: a fixation screen (400 $\mathrm{msec})$, Array $1(27 \mathrm{msec})$, a variable SOA, Array $2(27 \mathrm{msec})$, a retention interval $(1,000 \mathrm{msec})$, and the probe array (presented until the subject responded). We removed the visible grid because location information was not critical to the task.

Materials. Arrays 1 and 2 consisted of three solid circles $\left(1^{\circ} \times 1^{\circ}\right)$, and each circle was a unique color chosen randomly from a set of eight: red, green, blue, yellow, cyan, magenta, black, and white. The colored circles were presented at randomly selected locations within an invisible $5 \times 5$ grid matrix $\left(13^{\circ} \times 13^{\circ}\right)$ against a gray background, and there was no spatial overlap across arrays (see Figure 3 ).

On the probe display, a single circle was presented. Its color was either the same as one of the six colors previously shown (matching either Array 1 or Array 2, equally often) or a new color different from all six previously shown colors. The probe item was pre- sented at the same location as one of the memory colors, and when its color matched one of the memory colors, that color was in fact previously presented at that location. Location information was preserved on the probe screen to facilitate accuracy and simplify the decision process (see Sperling, 1960, on the topic of "whole report" vs. "partial report").

The subjects pressed the " $z$ " key on the computer keyboard if the probe matched one of the memorized colors, and the " " key if it did not match any of the memorized colors. As in Experiment 1, accuracy feedback and a score were provided after each trial.

The SOA between the two arrays was $27,127,227,527$, or $1,027 \mathrm{msec}$, with each SOA randomly assigned to one fifth of all trials. The subjects completed 20 practice trials and 480 experimental trials (divided into six blocks of 80 trials each).

Data analysis. We calculated $A^{\prime}$ at each SOA as a measure of the subject's memory sensitivity (Grier, 1971). This was necessary because the task involved detection of the memory signal ("match" or "not match"). We also measured percent correct and $d^{\prime}$, which led to patterns of results similar to those for $A^{\prime}$. $A^{\prime}$ was preferred as a measure of memory accuracy (Donaldson, 1993). ${ }^{3}$

\section{Results}

Figure 4 shows $A^{\prime}$ as a function of SOA and array, averaged across all the subjects.

A repeated measures ANOVA on array (Array $1 \mathrm{vs.}$ Array 2) and SOA (27 vs. 127 vs. 227 vs. 527 vs.

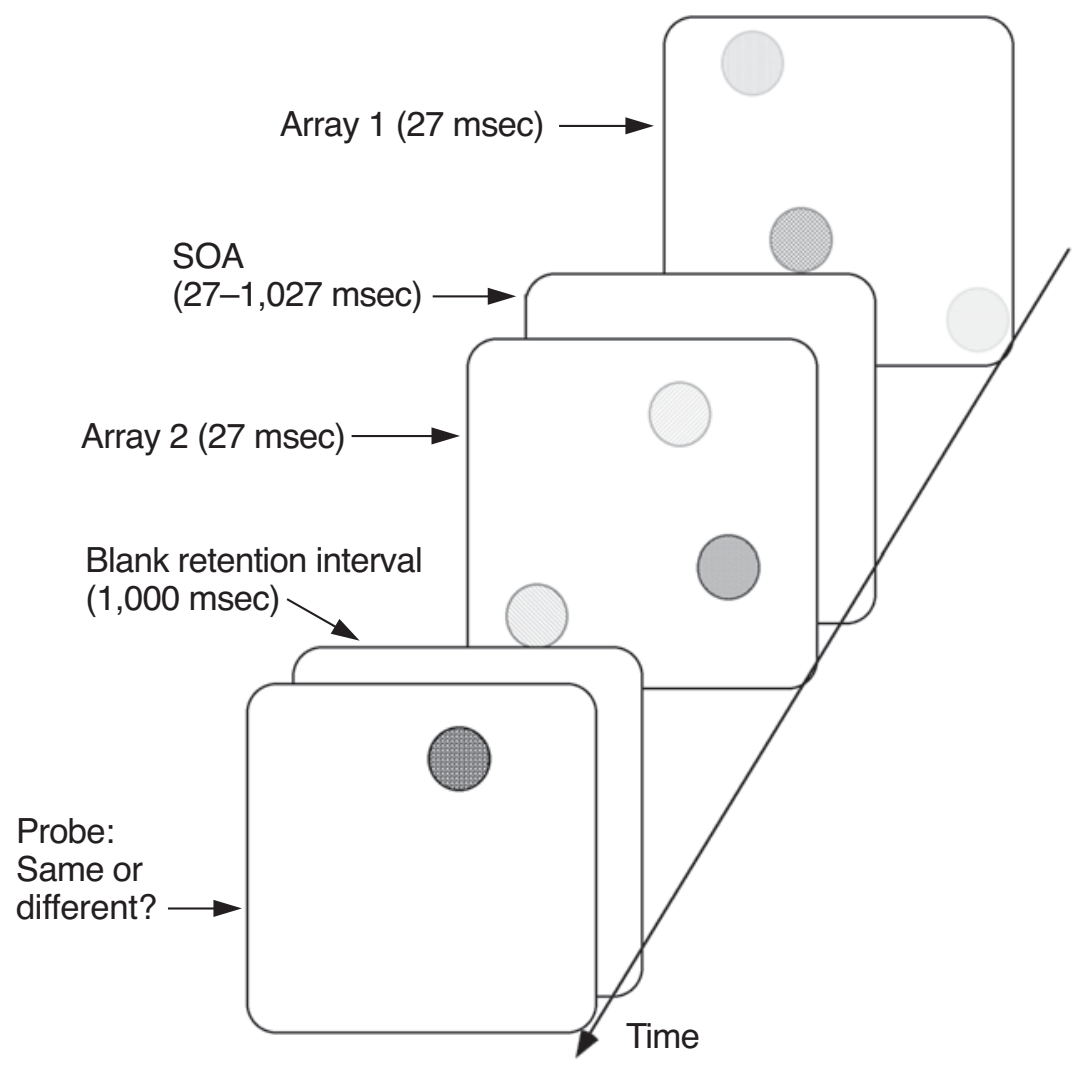

Figure 3. A schematic sample of the presentation sequence tested in Experiment 2. Subjects were told to remember the six colored dots presented in the two arrays and to judge whether a probe color matched a color in their memory. The actual stimuli were pure colors without texture; texture is added in this figure for illustrative purposes only. 
$1,027 \mathrm{msec}$ ) revealed a significant main effect of array $[F(1,11)=5.91, p<.05]$ and a significant interaction between array and SOA $[F(4,44)=2.83, p<.05]$, but the main effect of SOA was not significant $[F(4,44)<$ 1]. Thus, as in Experiment 1, Array 2 was better retained than Array 1, yet this effect was contingent on SOA. When the SOA was short $(27 \mathrm{msec})$, the two arrays were integrated in iconic memory and no difference was found in memory for the two arrays $[t(11)=0.70, p>.40]$. As the SOA increased, the two arrays were perceived as two temporal sequences, at which point memory for Array 2 started to show an advantage over memory for Array 1 $(p<.05)$ for the longest SOA. Pooled across the two arrays, the overall $A^{\prime}$ was not significantly influenced by SOA $(F<1)$, suggesting that VSTM for colors was comparable whether items were distributed into two temporal sequences or perceived as a single array.

In addition to $A^{\prime}$, we calculated Cowan's (2001) $K$ [i.e., (hit rate + correct rejection rate -1$) * N]$, separately for Arrays 1 and 2. The sum across the two arrays indicated the overall number of colors retained. The sum was 2.88 when the SOA was $27 \mathrm{msec}$ and 2.72 when it was $1,027 \mathrm{msec}$. These two values were not significantly different $[t(11)=.711, p>.49]$.

\section{Discussion}

As in Experiment 1, we observed VSTM for colors to have a constant capacity for simultaneous and sequential presentations. When Array 1 accuracy was low, Array 2 accuracy was high, and when Array 1 accuracy was intermediate Array 2 accuracy was also intermediate. This depicts a tradeoff, or competition, between the two arrays. The average of Array 1 and Array 2 represents the intermediate level, and since there is no significant variation in the average across SOAs, the capacity of VSTM

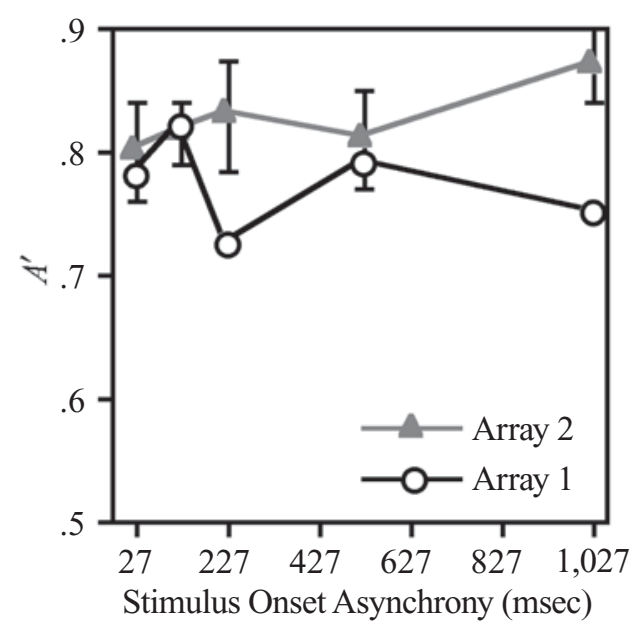

Figure 4. Results from Experiment 2. Subjects were shown three colors in each array. Memory for Array 2 was similar to that for Array 1 when SOA was short $(27 \mathrm{msec})$, but was much better at longer SOAs. The error bars show standard errors of the differences between Arrays 1 and 2. for color information appears to be constant. These results support the hypothesis that color VSTM capacity is independent of presentation mode. Sequential arrays compete for a single capacity in VSTM, so that one array is remembered at a cost to the other.

Together, the first two experiments provide strong support for the idea that a fixed capacity limits VSTM representation of spatial locations and colors independent of presentation mode. Such a representation shows a dramatic recency effect, with better memory for the trailing array than for the leading array.

\section{EXPERIMENT 3 VSTM for Different Features: Color and Orientation}

Experiment 3 extended the results from the first two experiments to conditions in which there were multiple types of visual information. In this experiment, visual information was divided not only temporally but also categorically, with colors in one array and orientations in the other. We asked whether temporal segregation enhances VSTM when it is accompanied by a clear categorical difference between information presented in the two temporal arrays.

Two conditions involving two types of features - color and orientation-were tested in this experiment. In the one-category condition, the two arrays contained the same type of features: three colors in each array, or three tilted lines in each array. In the two-category condition, the two arrays contained different types of features: three colors in one array and three tilted lines in the other. Although a total of six features - three from Array 1 and three from Array 2-were to be remembered in both conditions, the features were more heterogeneous in the two-category condition. On the basis of previous studies that show a VSTM advantage for heterogeneous features over homogeneous features (e.g., Olson \& Jiang, 2002; Wheeler \& Treisman, 2002; Xu, 2002a), we predicted that VSTM should be better for the two-category than for the one-category condition. In addition, the distribution of the two categories of features coincided with the temporal segregation: All the colors were in one temporal array, whereas all the orientations were in the other temporal array. The question was whether or not such a coincidence would lead to an advantage for sequentially presented arrays over simultaneously presented arrays.

We varied the SOA between the two arrays from 27 to $1,227 \mathrm{msec}$. On the basis of the results from the first two experiments, we predicted that in the one-category condition the overall VSTM accuracy should not be affected by the SOA. Of interest was whether in the two-category condition VSTM would be better for sequential than for simultaneous presentations. Figure 5 shows a schematic sample of a trial in the two-category condition.

\section{Method}

Subjects. Twenty-four subjects participated in this experiment for payment. 
Trial sequence. The subjects saw the following sequence on each trial: a blank screen $(400 \mathrm{msec})$, Array $1(27 \mathrm{msec})$, a variable SOA, Array $2(27 \mathrm{msec})$, a blank retention interval $(1,000 \mathrm{msec})$, and the probe array (presented until the subject responded).

Design. The one-category condition included two types of trials: color-color and orientation-orientation, for both Array 1 and Array 2. Three colored circles or three white tilted lines were presented in each array, and the two arrays of a single trial contained the same category of features. The colors were chosen from red, green, blue, yellow, magenta, cyan, black, and pink. The oriented white lines were chosen from $20^{\circ}$ tilt intervals between $0^{\circ}$ and $160^{\circ}$. As in Experiment 2 , all items were presented on a $5 \times 5$ invisible grid matrix $\left(16^{\circ} \times 16^{\circ}\right)$ against a gray background, with no spatial overlap between arrays. The probe array contained one item, which was presented at a previously occupied location: It either matched the item that was previously there (in color or in orientation) or it did not match any item. The color-color and orientation-orientation trials were randomly intermixed within each experimental block.

The two-category condition contained two types of trials: colororientation and orientation-color, for both Array 1 and Array 2. The probe array again contained one item from a previously occupied location. It was always the same type of item (colored circle or oriented line) as the item previously occupying that location; however, the item either matched one of the memorized items (in color or in orientation) or it did not match any item in memory. Color-orientation and orientation-color trials were randomly intermixed within an experimental block, as were one- and two-category conditions. As in Experiment 2, the subjects pressed the " $\mathrm{z}$ " key on the computer keyboard for a memory match, or the "/" key otherwise. $A^{\prime}$ was calculated.

The SOA between the two arrays was $27,227,527$, or $1,227 \mathrm{msec}$, with each SOA randomly assigned to one fourth of all trials. Feedback was provided after each trial. The subjects completed 20 practice trials and 384 experimental trials.

\section{Results}

We pooled across color and orientation displays because the patterns of results were qualitatively similar. Thus, color-color and orientation-orientation were averaged for the one-category condition, and color-orientation and orientation-color were averaged for the two-category condition. Memory performance, measured by $A^{\prime}$, is shown in Figure 6.

An ANOVA on array (Array 1 vs. Array 2), SOA (27 vs. 227 vs. 527 vs. 1,027 $\mathrm{msec}$ ), and category (one category vs. two categories) revealed a significant main effect of array $[F(1,23)=49.48, p<.001]$, with memory better for Array 2 than for Array 1. There was also a significant main effect of category, with memory for twocategory displays better than that for one-category displays $[F(1,23)=7.60, p<.011]$. This suggests that different types of features did not compete as much as same types of features for the capacity of VSTM. The

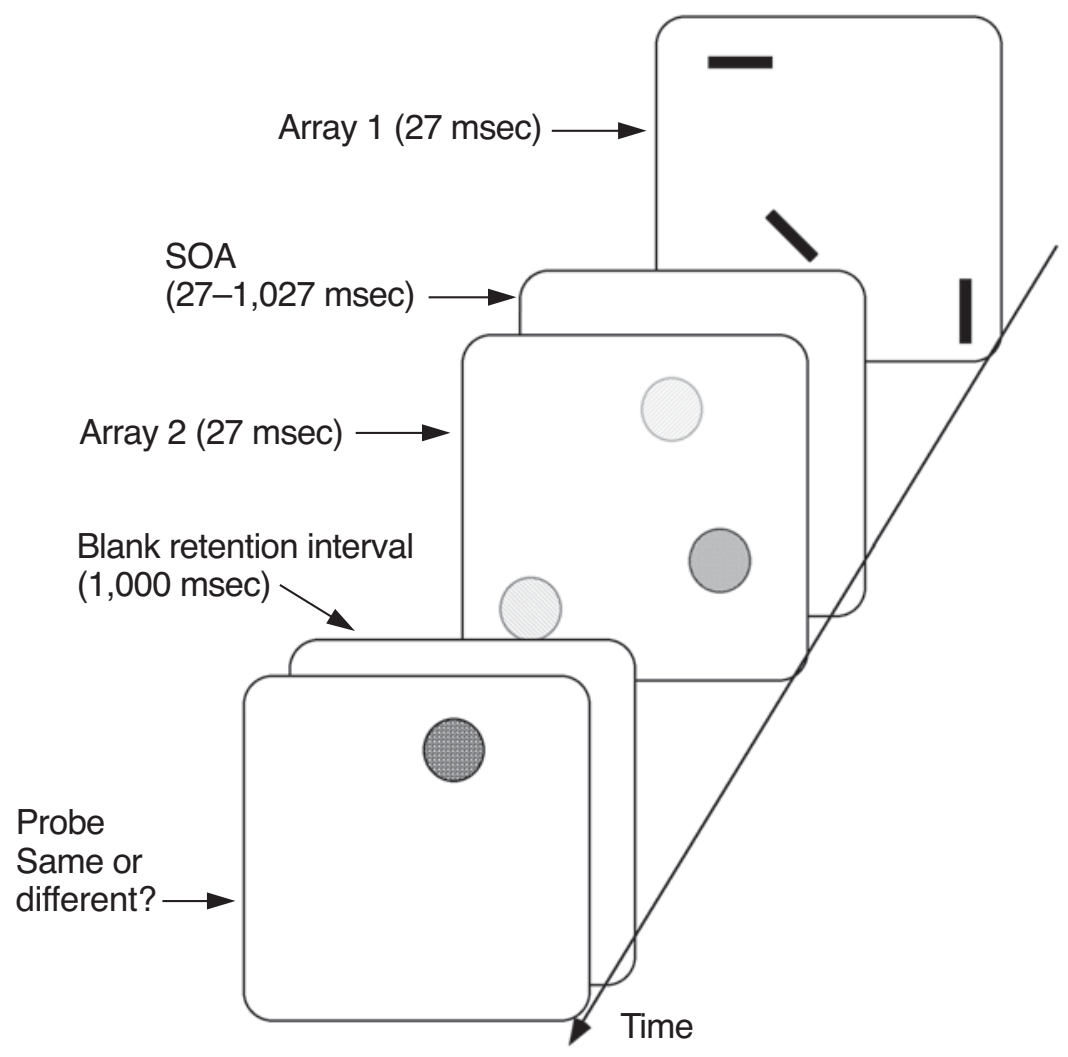

Figure 5. A schematic sample of the presentation sequence tested in Experiment 3. Subjects were told to remember colors and orientations presented in the two arrays and to judge whether a probe item matched one in their memory. The actual color stimuli were pure colors without texture. 


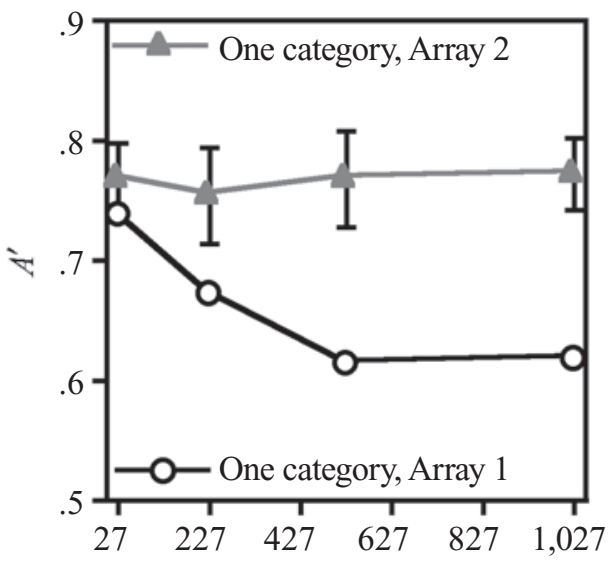

Stimulus Onset Asynchrony (msec)

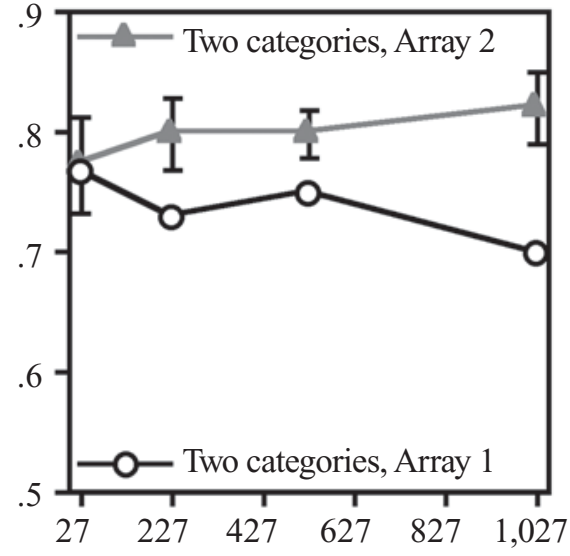

Stimulus Onset Asynchrony (msec)

Figure 6. Results from Experiment 3. In the one-category condition, the two arrays contained the same types of features (three colors or three orientations, in both arrays). In the two-category condition, one array contained three colors, and the other, three orientations. The overall VSTM performance, pooled across both arrays, was relatively constant across SOAs for both the one-category and two-category conditions. The error bars show standard errors of the differences between Arrays 1 and 2 .

main effect of SOA was not significant $[F(3,69)=1.30$, $p>.20]$. There was also a significant interaction between array and SOA $[F(3,69)=4.056, p<.01]$. This interaction existed because although memory was comparable for the two arrays at an SOA of $27 \mathrm{msec}$, it was much higher for Array 2 than for Array 1 as the SOA increased to $1,027 \mathrm{msec}$. None of the other interaction effects was significant [all $p \mathrm{~s}>.20$ ].

We also conducted separate ANOVAs on the onecategory and two-category trials. These revealed a main effect of array for both the one-category $[F(1,23)=$ $38.01, p<.001]$ and two-category $[F(1,23)=9.83, p<$ $.005]$ conditions. The interaction between array and SOA was significant in both the one-category condition $[F(3,69)=2.98, p<.037]$ and the two-category condition $[F(3,69)=3.02, p<.03]$. Pooled across the two arrays, memory performance was not significantly affected by SOA in either the one-category $[F(3,69)=2.10, p>$ $.10]$ or the two-category $[F(3,69)<1]$ condition. In the one-category condition, Cowan's $K$ averaged 4.12 and 3.56 for SOAs of $27 \mathrm{msec}$ and $1,027 \mathrm{msec}$, respectively $[t(23)=1.15, p>.20]$. In the two-category condition, Cowan's $K$ was 4.13 and 4.06 for SOAs of $27 \mathrm{msec}$ and $1,027 \mathrm{msec}$, respectively $[t(23)<1$, n.s.]. This suggests that sequential presentation did not enhance VSTM capacity in either condition.

\section{Discussion}

Even when temporal segregation coincided with the separation of features into two categories, with colors presented in one temporal array and orientations in the other, sequential presentation led to no memory advantage in comparison with simultaneous presentation. Instead, this experiment confirmed two conclusions. First, holding the number of features constant, VSTM for heterogeneous features was better than VSTM for homogeneous features. It was easier for people to retain three colors and three orientations than to retain six colors or six orientations, even though the colors and orientations were isolated features in both conditions. This advantage for heterogeneous features was relatively modestcertainly not enough to double memory capacity-but it was significant nonetheless (see also Olson \& Jiang, 2002). Second, distributing visual features into two temporal groups did not enhance the overall VSTM. Instead, the two visual arrays competed for a relatively constant VSTM capacity, with the trailing array winning the competition.

\section{EXPERIMENT 4 VSTM for Conjunction of Features}

In Experiment 4, we tested the conjunction of two features - color and orientation - in a single object instead of each feature in a separate object. Such chunking of features into single objects enhances the total number of features retained in VSTM (Luck \& Vogel, 1997). Here, we tested whether or not VSTM for conjunction of features was also insensitive to the presentation mode- that is, whether the total number of objects stored in VSTM would be influenced by presentation mode.

In this experiment, we used a design similar to that of the previous three, including three conditions. In the three-single-features condition, each array contained three features (e.g., three colors in Array 1 and three colors in Array 2, or three orientations in Array 1 and three orientations in Array 2). In the six-single-features condition, each array contained six features (e.g., six colors in Array 1 
and six colors in Array 2). In the six-conjoined-features condition, each array contained three colored lines, for a total of six features. On the basis of the findings of previous studies, we expected performance to be higher in the three-single-features condition than in the six-singlefeatures condition, because there were fewer features to be retained in the former. We also expected performance to be better in the six-conjoined-features than in the sixsingle-features condition because of object-based chunking. Of interest was whether sequential presentation of images would have similar effects on conjunction of features (the six-conjoined-features condition) as on simple features (the three- and six-single-features conditions).

\section{Method}

Subjects. Twenty-four subjects participated for payment.

Trial sequence. The subjects saw the following sequence: a fixation screen ( $400 \mathrm{msec})$, Array 1 ( $27 \mathrm{msec})$, a blank screen (variable SOA), Array 2 (27 msec), a blank retention interval (1,000 msec), and the probe array (presented until the subject responded).

There were three feature conditions: the three-single-features, six-single-features, and six-conjoined-features conditions, named according to the number of features presented in each array. The three conditions were presented in a randomly mixed order. In the three-single-features condition, each array contained three colors on half of the trials and three orientations on the other half of the trials. In the six-single-features condition, each array contained six colors on half of the trials and six orientations on the other half of the trials. Finally, in the six-conjoined-features condition, each array contained three colored, oriented lines (for a total of six features). No color or orientation was repeated within a single trial. Items were presented at randomly selected locations from an invisible $5 \times 5$ grid matrix. The two arrays did not overlap in locations.

The probe arrays for all trials showed two items, one of which was identical to an object from either Array 1 or Array 2 (randomly and evenly divided) and the other of which was nearly identical to an object from either Array 1 or Array 2, but with one feature altered. The new feature did not match any features presented in Arrays 1 and 2 . A small " 1 " or " 2 " was presented slightly below the probe items, randomly assigned and uncorrelated with the array condition. The subjects identified which of the items matched an item in their memory perfectly by pressing the " 1 " or the " 2 " key on the keyboard, in correspondence with the digit below the matching probe. Accuracy feedback was provided immediately after each trial.

The SOA between the two arrays was $27,227,527$, or $1,227 \mathrm{msec}$, evenly and randomly mixed. The subjects completed 20 practice trials and 432 experimental trials. Accuracy was recorded.

\section{Results and Discussion}

We calculated percent correct for each array in each SOA condition. Figure 7 shows the group means.

A repeated measures ANOVA on feature condition (three single features vs. six single features vs. six conjoined features), array (Array 1 vs. Array 2), and SOA (27 vs. 227 vs. 527 vs. $1,227 \mathrm{msec}$ ) revealed significant main effects of feature $[F(2,46)=7.41, p<.002]$ and array $[F(1,23)=39.31, p<.001]$ but no main effect of SOA $[F(3,69)<1]$. The interaction between array and SOA was highly significant $[F(3,69)=8.60, p<.001]$. Memory was similar for the two arrays when the SOA was $27 \mathrm{msec}(p>.20)$ but became different as the SOA increased. Accuracy was much higher for Array 2 than for Array 1 when the SOA between the two arrays was $1,227 \mathrm{msec}(p<.005)$. No other interaction effect was significant [all $p \mathrm{~s}>.20]$.

Planned contrasts showed that accuracy was significantly higher in the three-single-features than in the sixsingle-features condition $[F(1,23)=12.51, p<.002]$. This was not surprising, since there were more features to be remembered in the latter. Memory for the sixconjoined-features condition was comparable with that for the three-single-features condition $[F(1,23)=2.28$, $p>.15]$, suggesting that when the number of objects is held constant, presentation of multiple features in a single object did not impair VSTM performance significantly. Finally, memory for the six-conjoined-features condition was significantly better than that for the sixsingle-features condition $[F(1,23)=5.05, p<.034]$,
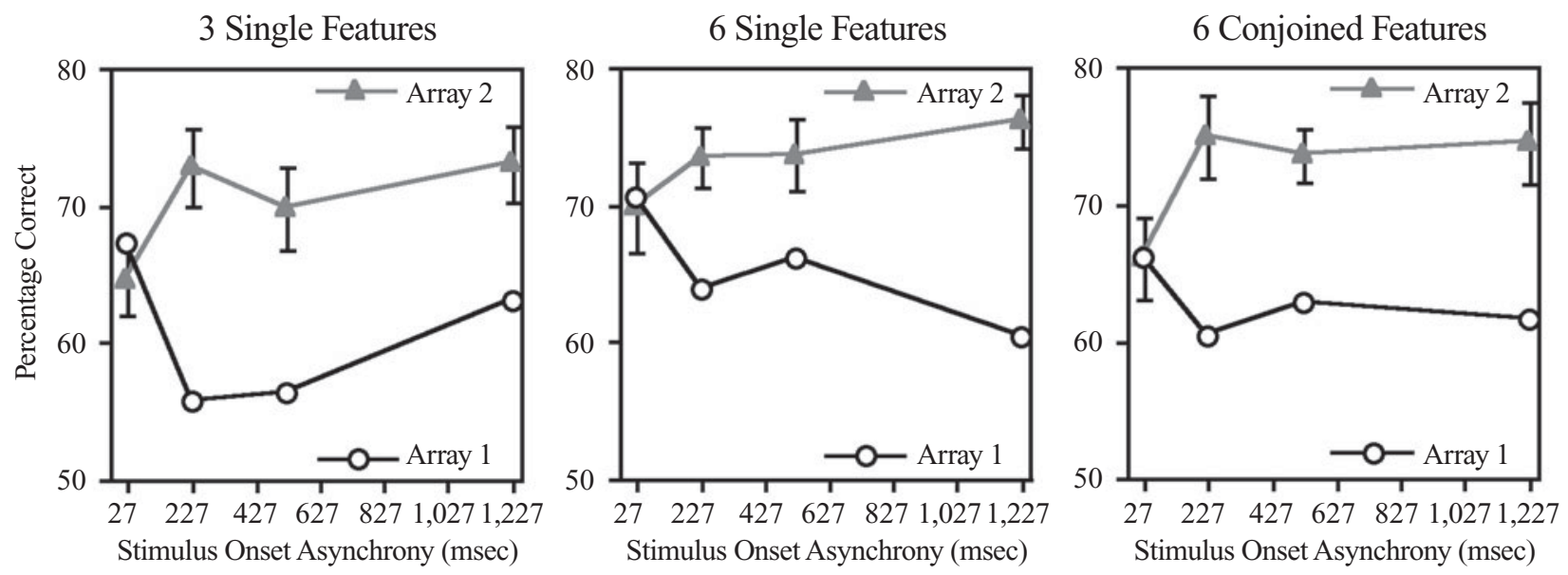

Figure 7. Results from Experiment 4. Whether each array contained three single features, six single features, or six conjoined features, VSTM for Array 2 was much better than that for Array 1. Pooled across the two arrays, accuracy was relatively constant across all SOAs, suggesting a constant overall capacity. The error bars show standard errors of the differences between Arrays 1 and 2. 
showing that when the number of features is held constant, VSTM was better when features were conjoined than when they were isolated.

In all three conditions, there was a main effect of array, with better accuracy for Array 2 than for Array 1 (all $p \mathrm{~s}<.01)$. However, the main effect of SOA was not significant in any condition, suggesting that VSTM was relatively stable whether items were perceived as presented simultaneously (i.e., when $\mathrm{SOA}=27 \mathrm{msec}$ ) or sequentially (e.g., when SOA = 1,227 msec). Thus, whether single features or a conjunction of features were to be remembered, VSTM was relatively stable across the different SOAs.

\section{GENERAL DISCUSSION}

In four experiments, we tested VSTM for spatial locations, colors, orientations, and the conjunction of colors and orientations. Items were presented in two arrays separated by a variable SOA. When the SOA was $27 \mathrm{msec}$, the two arrays were integrated in iconic memory, resulting in a percept of a simultaneously presented array. As the SOA became longer (e.g., >1,000 msec), the two arrays were clearly perceived as being sequential. Surprisingly, pooled across the two arrays, VSTM for all items was comparable across all SOAs, whether items were perceived as simultaneously presented or temporally segregated. This is a surprising finding in light of previous studies that have shown an increase in VSTM capacity when the to-be-remembered information is separated into two depth planes (Xu \& Nakayama, 2003) or two spatial containers (Feigenson, Carey, \& Hauser, 2002). Chunking information into two temporal groups does not double the total amount of information retained in VSTM. Instead, the two temporal arrays compete with each other for retention in VSTM, with the second array gaining an advantage in the competition. This pattern is observed independent of the kinds of features presented in each array (locations, colors, orientations, or a conjunction of colors and orientations). No advantage is seen even when the temporal segregation coincides with categorical separations, with one type of feature (e.g., color) in one array and another type of feature (e.g., orientation) in the other. Thus, although spatial grouping cues can expand the total amount of information stored in VSTM, temporal grouping cues have nearly no effect on the total capacity of VSTM.

The insensitivity of VSTM to temporal grouping is not a result of VSTM's automatically integrating the sequential displays in such a way that the displays are converted into a large array of simultaneously presented items. A recent study shows that under the current testing conditions, subjects retain separate representations for each array at intermediate and long SOAs (Jiang \& Kumar, 2004). A fixed total VSTM capacity is thus distributed across sequential representations, with a larger allotment to the trailing array. Unpublished data from our lab show that this conclusion is not restricted to in- formation distributed across only two arrays: Dividing items into three arrays (each having two items) or six arrays (each having one item) leads to the same overall memory accuracy as when all six items are presented simultaneously. Clearly, the overall capacity of VSTM does not expand on the dimension of time.

Given its limitation in capacity, the visual system has settled into prioritizing the trailing array. This prioritization was consistently seen in all conditions, even though our subjects were always instructed to give equal priority to both arrays. This prioritization may occur because the second array acts as a mask on the first, in different ways across different SOAs: When the SOA is intermediate (e.g., 300-500 msec), the second array interrupts the consolidation of the first, producing "conceptual" masking - a phenomenon supported by VSTM studies employing similar paradigms (see, e.g., Brockmole et al., 2002; Di Lollo \& Moscovitch, 1983). At a much longer SOA (e.g., $>1,000 \mathrm{msec}$ ), the first array is most likely already consolidated (Vogel et al., in press). Array 2 pushes Array 1 outside of VSTM, resulting in rapid forgetting. This competition between memory items was first demonstrated in studies of short-term interference (see, e.g., Waugh \& Norman, 1965), and the observed pushing out of the leading array may contribute to our illusory sense of a continuous world (see also O'Regan, 1992). Because our VSTM is dominated by the most recent information, with earlier information pushed out, we do not have sufficiently clear representations to compare information across time. This observation may also contribute to our inability to find changes in natural viewing: Very little of the preceding displays is retained, so comparison across displays is difficult.

In summary, we find that VSTM for locations, surface features, and conjunctions has a relatively constant capacity limit that is independent of how items are presented. Separating information into two sequential arrays leads to no memory advantage. Instead, the two arrays compete for retention in VSTM, with the trailing array gaining a clear advantage in the competition. We suggest that VSTM for sequentially presented items shares the same limited capacity as VSTM for simultaneously presented items.

\section{REFERENCES}

Alais, D., Blake, R., \& LeE, S. H. (1998). Visual features that vary together over time group together over space. Nature Neuroscience, $\mathbf{1}$, 160-164.

Alvarez, G. A., \& Cavanagh, P. (2004). The capacity of visual short term memory is set both by visual information load and by number of objects. Psychological Science, 15, 106-111.

Baddeley, A. D. (1986). Working memory. Oxford: Oxford University Press, Clarendon Press.

Blake, R., \& YANG, Y. (1997). Spatial and temporal coherence in perceptual binding. Proceedings of the National Academy of Sciences, 94, 7115-7119.

Broadbent, D. E., \& Broadbent, M. H. P. (1981). Recency effects in visual memory. Quarterly Journal of Experimental Psychology, 33A, $1-15$.

Brockmole, J. R., Irwin, D. E., \& WANG, R. F. (2003). The locus of 
spatial attention during the temporal integration of visual memories and visual percepts. Psychonomic Bulletin \& Review, 10, 510-515. Brockmole, J. R., Wang, R. F., \& Irwin, D. E. (2002). Temporal integration between visual images and visual percepts. Journal of Experimental Psychology: Human Perception \& Performance, 28, 315334.

Cowan, N. (2001). The magical number 4 in short-term memory: A reconsideration of mental storage capacity. Behavioral \& Brain Sciences, 24, 87-114.

Delvenne, J.-F., \& BRuyer, R. (2004). Does visual short-term memory store bound features? Visual Cognition, 11, 1-27.

DI LoLLO, V. (1980). Temporal integration in visual memory. Journal of Experimental Psychology: General, 109, 75-97.

Di Lollo, V., \& Moscovitch, M. (1983). Perceptual interference between spatially separate sequential displays. Canadian Journal of Psychology, 37, 414-428.

Donaldson, W. (1993). Accuracy of $d^{\prime}$ and $A^{\prime}$ as estimates of sensitivity. Bulletin of the Psychonomic Society, 31, 271-274.

Feigenson, L., Carey, S., \& Hauser, M. (2002). The representations underlying infants' choice of more: Object files versus analog magnitude. Psychological Science, 13, 150-156.

GrIER, J. B. (1971). Nonparametric indexes for sensitivity and bias: Computing formulas. Psychological Bulletin, 75, 424-429.

IRWIN, D. E. (1991). Information integration across saccadic eye movements. Cognitive Psychology, 23, 420-456.

JiANG, Y. (2004). Time window from visual images to visual short-term memory: Consolidation or integration? Experimental Psychology, $\mathbf{5 1}, 45-51$.

Jiang, Y., Chun, M. M., \& Marks, L. E. (2002). Visual marking: Selective attention to asynchronous temporal groups. Journal of Experimental Psychology: Human Perception \& Performance, 28, 717-730.

Jiang, Y., Chun, M. M., \& Olson, I. R. (2004). Perceptual grouping in change detection. Perception \& Psychophysics, 66, 446-453.

JiANG, Y., \& KUMAR, A. (2004). Visual short-term memory for two sequential arrays: One integrated representation or two separate representations? Psychonomic Bulletin \& Review, 11, 495-500.

JiANG, Y., KUMAR, A., \& VICKERY, T. J. (2005). Integrating sequential arrays in visual short-term memory. Experimental Psychology, 52, 39-46.

Jiang, Y., Olson, I. R., \& CHUN, M. M. (2000). Organization of visual short-term memory. Journal of Experimental Psychology: Learning, Memory, \& Cognition, 26, 683-702.

Joliceler, P., \& Dell'ACQua, R. (1998). The demonstration of shortterm consolidation. Cognitive Psychology, 36, 138-202.

LeE, D., \& Chun, M. M. (2001). What are the units of visual short-term memory, objects or spatial locations? Perception \& Psychophysics, 63, 253-257.

LeE, S. H., \& BlaKe, R. (1999). Visual form created solely from temporal structure. Science, 284, 1165-1168.

Livingstone, M., \& Hubel, D. (1988). Segregation of form, color, movement, and depth: Anatomy, physiology, and perception. Science, 240, 740-749.

Loftus, G. R., Duncan, J., \& Gehrig, P. (1992). On the time course of perceptual information that results from a brief visual presentation. Journal of Experimental Psychology: Human Perception \& Performance, 18, 530-549.

LOGIE, R. H. (1995). Visuo-spatial working memory. Hillsdale, NJ: Erlbaum.

LUCK, S. J., \& Vogel, E. (1997). The capacity of visual working memory for features and conjunctions. Nature, 309, 279-281.

Macmillan, N. A., \& Creelman, C. D. (1991). Detection theory: A user's guide. New York: Cambridge University Press.

MishKin, M., \& UnGERLEIDER, L. G. (1983). Contribution of striate inputs to the visuospatial functions of parieto-preoccipital cortex in monkeys. Behavior \& Brain Research, 6, 57-77.

OLSON, I. R., \& JiANG, Y. (2002). Is visual short-term memory object based? Rejection of the "strong-object" hypothesis. Perception \& Psychophysics, 64, 1055-1067.

O'Regan, J. K. (1992). Solving the "real" mysteries of visual perception: The world as an outside memory. Canadian Journal of Psychology, 46, 461-488.
Pashler, H. (1988). Familiarity and visual change detection. Perception \& Psychophysics, 44, 369-378.

PhiLlips, W. A. (1974). On the distinction between sensory storage and short-term visual memory. Perception \& Psychophysics, 16, 283-290.

PotTer, M. C. (1976). Short-term conceptual memory for pictures. Journal of Experimental Psychology, 2, 509-522.

Potter, M. C., Staub, A., \& O'Connor, D. H. (2002). The time course of competition for attention: Attention is initially labile. Journal of Experimental Psychology: Human Perception \& Performance, 28, 1149-1162.

RAFFone, A., \& Wolters, G. (2001). A cortical mechanism for binding in visual working memory. Journal of Cognitive Neuroscience, 13, 766-785.

SPERLING, G. (1960). The information available in brief visual presentation. Psychological Monographs: General \& Applied, 74, 1-29.

Usher, M., \& DoNnelly, N. (1998). Visual synchrony affects binding and segmentation in perception. Nature, 394, 179-182.

Vogel, E. K., Woodman, G. F., \& LucK, S. J. (2001). Storage of features, conjunctions, and objects in visual working memory. Journal of Experimental Psychology: Human Perception \& Performance, 27, 92-114.

Vogel, E. K., Woodman, G. F., \& Luck, S. J. (in press). The time course of consolidation in visual working memory. Journal of Experimental Psychology: Human Perception \& Performance.

WALKer, P., \& Davies, S. J. (2003). Perceptual completion and objectbased representations in short-term visual memory. Memory \& Cognition, 31, 746-760.

Waugh, N. C., \& Norman, D. A. (1965). Primary memory. Psychological Review, 72, 89-104.

WheELER, M. E., \& Treisman, A. M. (2002). Binding in short-term visual memory. Journal of Experimental Psychology: General, 131, 48-64.

Woodman, G. F., Vecera, S. P., \& Luck, S. J. (2003). Perceptual organization influences visual working memory. Psychonomic Bulletin \& Review, 10, 80-87.

XU, Y. (2002a). Encoding color and shape from different parts of an object in visual short-term memory. Perception \& Psychophysics, 64, $1260-1280$.

XU, Y. (2002b). Limitations of object-based feature encoding in visual short-term memory. Journal of Experimental Psychology: Human Perception \& Performance, 28, 458-468.

Xu, Y., \& Nakayama, K. (2003). Placing objects at different depths increases visual short-term memory capacity [Abstract]. Journal of $\mathrm{Vi}$ sion, 3, 27a. Available at http://journalofvision.org/3/9/27/.

\section{NOTES}

1. To ensure that the shortest SOA $(27-\mathrm{msec})$ condition is truly a perceptually simultaneous presentation, we tested 12 subjects in two pilot experiments, one on VSTM for colors and one on VSTM for locations. These subjects were tested in three conditions: (1) simultaneous, in which all items were presented simultaneously; (2) near simultaneous, in which items were divided evenly into Arrays 1 and 2, with an SOA of $27 \mathrm{msec}$ between the two arrays; and (3) sequential, in which the SOA between the two arrays was $1,200 \mathrm{msec}$. Results showed identical memory performance for the simultaneous and near simultaneous conditions. These data suggest that the shortest SOA condition was representative of simultaneous presentation conditions. For simplicity, we refer to the "SOA $=27 \mathrm{msec}$ " condition as the simultaneous condition in the present study.

2. We thank Steven Luck for raising this point.

3. Whether $A^{\prime}$ or $d^{\prime}$ should be calculated is controversial. Macmillan and Creelman (1991) recommended $d^{\prime}$ for perceptual detection tasks, whereas Donaldson (1993) recommended $A^{\prime}$ for memory sensitivity. The dissatisfaction with these measures pushed us to move into a twoalternative forced choice task in Experiments 2 and 3, which obviated signal detection issues.

(Manuscript received March 31, 2004; revision accepted for publication July 21, 2004.) 